\title{
Tightly regulated gene expression in human hematopoietic stem cells after transduction with helper-dependent Ad5/35 vectors
}

\author{
Hongje Wang ${ }^{1}$, Hua Cao ${ }^{1}$, Martin Wohlfahrt ${ }^{3}$, Hans-Peter Kiem ${ }^{3}$, and André Lieber ${ }^{1,2}$ \\ 1 Division of Medical Genetics, Department of Medicine, University of Washington, Seattle, WA \\ 2Department of Pathology, University of Washington, Seattle, WA \\ 3 Fred Hutchinson Cancer Research Center, Seattle, WA
}

\begin{abstract}
Objective-Inducible and transient expression of transcription factors, growth factors, or mitogenic factors can be used to influence proliferation or differentiation of hematopoietic progenitor/stem cells (HSCs). Furthermore, transient expression of proteins with site-specific endonuclease activity, potentially, can support targeted integration of exogenous transgenes into specific sites in the genome, a task that is currently a focus in development of gene therapy vectors.
\end{abstract}

Methods-We constructed a set of helper-dependent adenovirus (Ad) vectors with serotype 35 fiber knob domains (HD-Ad5/35) which can efficiently transduce human CD34+ cells, particularly subsets with potential stem cell capacity. These vectors contain Tet-inducible expression systems that were shielded by insulators and transcription stop signals to minimize unspecific interference by transcriptional elements present in viral and stuffer DNA. We compared two vectors, containing a fusion between the Krüppel-associated box (KRAB) domain and the tetracycline repressor (HDAd5/35.Tet-1) or an autoregulated rtTA (HD-Ad5/35.Tet-2) for regulated transgene expression in Mo7e cells, a model for HSC, and primary human $\mathrm{CD} 34^{+}$cells.

Results-HD-Ad5/35.Tet-1 conferred lower background expression than HD-Ad5/35.Tet-2, although levels of induced gene expression were higher for HD-Ad5/35.Tet-2. In CD34+ cells, while HD-Ad5/35.Tet-1 allowed for activated gene expression in all transduced cells, induced gene expression from HD-Ad5/35.Tet- 2 was restricted to a small subset of CD34 ${ }^{+}$cells. Importantly, clonogeneic assays and repopulation studies in NOD/SCID mice showed that both HD-Ad5/35.Tet-1 and -2 vectors mediated induced gene expression in primitive hematopoietic cells. These studies also confirmed that transduction of $\mathrm{CD} 34^{+}$cells with HD-Ad5/35 vectors is not associated with cytotoxity, a problem observed with first-generation Ad5/35 vectors.

Conclusions-Both HD-Ad5/35 vector expression systems confer tightly regulated, transient transgene expression in human HSC.

\section{Keywords}

gene therapy; adenovirus; human hematopoietic stem cells

Corresponding author: Dr. André Lieber MD, PhD, University of Washington, Division of Medical Genetics, Box 357720, Seattle, WA 98195, phone: (206) 221-3973, fax: (206) 685-8675, email: lieber00@u.washington.edu.

Publisher's Disclaimer: This is a PDF file of an unedited manuscript that has been accepted for publication. As a service to our customers we are providing this early version of the manuscript. The manuscript will undergo copyediting, typesetting, and review of the resulting proof before it is published in its final citable form. Please note that during the production process errors may be discovered which could affect the content, and all legal disclaimers that apply to the journal pertain. 


\section{Introduction}

Transient and inducible expression of transgenes in human hematopoietic stem cells from nonintegrating gene transfer vectors is instrumental in studying the role of specific proteins in stem cell biology and achieving targeted genome modifications, including site-specific gene addition, deletion, or replacement. Our group is specifically interested in targeting transgene expression cassettes to specific sites in the genome of human hematopoietic stem cells (HSCs) by transient expression of proteins with site-specific endonuclease activity, such as the AAV Rep78 protein [1] and sequence-specific zinc-finger nucleases [2]. Gene transfer into HSCs by transfection or electroporation is hampered by low efficiency and high cytotoxicity. Nonintegrating lentivirus vector have shown promise for transient expression of transgenes in HSC but suffer from relatively low transduction and expression levels [3]. We have focused on nonintegrating adenovirus (Ad) vectors for gene transfer into human HSC. Because commonly used species C Ad serotype 5-based vectors do not efficiently transduce HSC, we developed chimeric Ad5 vectors that carry fibers from species B Ad serotype 35 (Ad5/35) [4]. These vectors infect cells through CD46, a protein whose expression is upregulated in undifferentiated cells, including HSC [5]. Previous studies showed that Ad5/35 vectors efficiently infect human $\mathrm{CD} 34^{+}$cells, particularly subsets with potential stem cell activity. For example, at an MOI of 10pfu/cell, Ad5/35 transduced the majority of CD34 $/ \mathrm{c}-\mathrm{Kit}^{+}$[4], CD34 ${ }^{+} / \mathrm{CD}^{-} 8^{-}, \mathrm{CD}^{-} 4^{-} / \mathrm{Lin}^{-}$, and Hoechst 33342 dye-negative side population (SP) cells [6]. Furthermore, Ad5/35 vectors allowed for efficient gene transfer into non-dividing CD $34^{+}$cells [7], which is important as most of the repopulating HSCs are quiescent. These transduction studies were done with first-generation (E1-/E3-deleted) Ad5/35 vectors and the readout was transgene expression 24 to 48 hours after infection. However, first-generation Ad vectors contain viral genes which can potentially be expressed in transduced cells and affect cell viability or function [8]. This problem can be addressed by using helper-dependent (HD) Ad5/35 vectors that are devoid of all viral genes. Production of these vectors requires an Ad5/35 helper virus whose packaging can be controlled by Cre/lox recombination [9]. In previous studies, we showed that transient gene expression from HD-Ad5/35 vectors in CD34 ${ }^{+}$cells was as efficient as with first-generation Ad5/35 vectors [10,11].

We plan to use HD-Ad5/35 vectors for transient expression of proteins with sequence-specific nuclease activity in $\mathrm{CD} 34^{+}$cells. Such vectors should lack endonuclease expression in producer cells (293 cells) and allow for inducible expression in HSC. However, the incorporation of inducible expression systems into Ad vectors is problematic because of interference by viral sequences. Ad ITRs have promoter activity and the Ad packaging signal overlaps with the E1 A enhancer $[12,13]$. The presence of these regulatory elements, that cannot be removed from the vector backbone, affects tissue specific expression [14,15], and also causes high background expression levels without induction when Tet-on or Tet-off expression systems are incorporated into first-generation [16] or HD Ad5 vectors [17]. We and others have shown that this problem can be addressed by incorporating insulators [18-20] and/or polyadenylation signals [21] into the vector genome.

In this study, we constructed HD-Ad5/35 vectors containing two alternative Tet-inducible expression systems that were shielded by insulators and transcription stop signals, and tested them in Mo7e cells and primary human $\mathrm{CD} 34^{+}$cells.

\section{Material and Methods}

\section{Ad vectors}

All shuttle plasmids were based on the cosmid vector pWE15 (Stratagene, La Jolla, CA). The ClaI site and NheI site in pWE15 were deleted. pWE-HCA was constructed by inserting the Ad5 5' ITR and packaging signal linked to a 8-kb PmeI/EcoRV fragment derived from pHCA 
[10], a 11.6-kb NheI/PacI pHCA fragment, and the Ad5 3' ITR into pWE15. A unique ClaI site was introduced between these two pHCA fragments. pWE-HCA also contains two FseI sites distal of the Ad ITRs. Next, a 11.8-kb stuffer DNA fragment (a combined fragment from human $\beta$-globin LCR and pHCA) was inserted into the BgIII site of pWE15-HCA to create a plasmid that has an optimal size for packaging into phage. For packaging the Gigapack III Plus Packaging Extract Kit (Stratagene, La Jolla, CA) was used. pHD-Ad.Tet-1 contains the Tetinducible GFP expression cassette from pLVPT-tTRKRAB [22]. We first constructed pBStTRKRAB. The TetO fragment was excised from pLVPT-tTRKRAB by XhoI/ClaI. The pgkGFP-IRES-TetRKRAB cassette was excised from pLVPT-tTRKRAB by ClaI/EcoRV.

Chromatin insulator cHS-4 was excised from pSLJC [18]. Polyadenylation signals from the hGH gene and SV40 large T antigen were PCR amplified from pCMV-MCS (Stratagene, La Jolla, CA) and pcDNA3.1 (Invitrogen, Carlsbad, CA), respectively using the following primers: Primers for the SV40pA Forward:ATGAGCTCAATCGATTCTAGACTCGAGAGTCGACACCCCTCGACCTC AT; Reverse: TAAGGGCCCAATCGATTACTGCAGAACTAGTATCCAGACATGAT Primers for hGHpA Forward: GACTGGTACCAAAGCTTGATCTACGGGTGGCA; Reverse: CTGAGGTACCGCTAGCAGAATTCGCTCGGTCCGCACGT A 1.8-kb stuffer DNA was PCR amplified from bacteriophage Lambda DNA and inserted between the TetO and PGK promoter. The following primers were used for stufferDNA : Forward, 5'TGCAGTCGACAGAATTCGTCCTTTCCGGCAATCAGG-3', and Reverse, 5' ACGTGTCGACACTAGTGCCTCCTTCGTGAAGACAA-3'. The expression cassettes flanked by cHS4 and polyA signals was excised from pBS-tTRKRAB and inserted into ClaI site of pWE-HCA to make pHD-Ad.Tet-1.

pHD-Ad.Tet-2 was constructed using TREAutoR3 [23]. We first constructed pBSTREAutoR3. The pminCMV-GFP-IRES-rtTA3 cassette was excised from pTREAutoR3 by $\mathrm{ClaI} / \mathrm{NheI}$ and inserted into the ClaI site of pWE-HCA. To bring the final HD-Ad genome to a size that is optimal for packaging into adenovirus capsids, a $2.5-\mathrm{kb}$ stuffer DNA from phage lambda DNA was inserted in front of the left cHS-4 element. The following primers were used for stuffer DNA: Forward, 5'-GACTTCTAGACGGCATACAGCAACAACATGG-3', and Reverse, 5'-CTGAGCTAGCAGCGTGAACGTCAGGATCACG-3'.

To generate HD-Ad vectors HD-Ad5/35-Tet-1 and HD-Ad5/35.Tet-2, viral genomes were released from corresponding plasmids by FseI digestion. HD-Ad vectors were rescued, amplified, and purified as described earlier [24]. Briefly, C7.Cre cells, which express Cre recombinase to prevent packaging of helper virus genomes by excising LoxP flanked packaging signals, were used to propagate HD-Ad5/35 virus. Cells were transfected with linearized HD plasmids and the following day infected with Ad5/35 helper virus at an MOI of $20 \mathrm{PFU} /$ cell. Cell lysates were serially passaged in C7.cre cells and Ad5/35 helper virus was added at each step (MOI $20 \mathrm{PFU} / \mathrm{cell}$ ). After several rounds of amplification, virus was purified by cesium chloride gradient centrifugation. Ad genome concentrations were determined by both quantitative Southern blotting and qPCR as described earlier [24].

\section{Cells}

Culture medium (DMEM, IMDM, and RPMI 1640) were purchased from GIBCO/BRL (Gaithersburg, MD, USA). Fetal calf serum (FCS) was from Hyclone (Logan, UT, USA). 293 (human embryonic kidney; Microbix, Toronto Canada) and C7-cre cells [25] were maintained in DMEM, supplemented with 10\% FCS, 2mM L-glutamine and 1X penicillin-streptomycin solution (Invitrogen, Carlsbad, CA). For C7-cre cells, $0.5 \mathrm{mg} / \mathrm{ml} \mathrm{G418} \mathrm{and} 100 \mu \mathrm{g} / \mathrm{ml}$ hygromycin was added to maintain Cre, Ad polymerase, and Ad pTP expression [26]. Mo7e cells were maintained in RPMI 1640 medium containing 10\% FCS, 2mM L-glutamine, Pen/ Strep, and $0.1 \mathrm{ng} / \mathrm{ml}$ of granulocyte-macrophage colony stimulating factor (Amgen, Seattle, 
WA). Primary human CD34+-enriched cells were purified cord blood PBMC by MiniMACS $\mathrm{LS}^{+}$separation columns (Miltenyi Biotec, Auburn, CA) using the CD34 -progenitor cell isolation kit (StemCell Technologies, Vancouver, BC, Canada), according to the manufacturers protocol. Aliquots of cells were stored in liquid nitrogen. Sixteen hours before the experiment, $\mathrm{CD} 34^{+}$cells were recovered from frozen stocks and incubated overnight in IMDM, supplemented with $20 \% \mathrm{FCS}, 10^{-4} \mathrm{M} \beta$-mercaptoethanol, $100 \mu \mathrm{g} / \mathrm{ml}$ DNase I, $2 \mathrm{mM}$ glutamine, $10 \mathrm{U} / \mathrm{ml} \mathrm{IL}-3,50 \mathrm{ng} / \mathrm{ml}$ stem cell factor, $50 \mathrm{ng} / \mathrm{ml} \mathrm{Flt3L}$ and $10 \mathrm{ng} / \mathrm{ml}$ thrombopoietin. The purity of $\mathrm{CD} 34^{+}$cell preparations was verified by flow cytometry and was consistently greater than $90 \%$. Cell viability was measured by PI flow cytometry.

\section{Colony assays}

For colony forming unit assay, 3000 infected CD34+ ${ }^{+}$cells were cultivated with $1 \mathrm{ml}$ MethoCult ${ }^{\mathrm{TM}}$ GF H4434 medium (StemCell Technologies) in a humidified atmosphere of 5\% $\mathrm{CO}_{2}$ at $37^{\circ} \mathrm{C}$ in the presence of the following cytokines: (IL-3 50U/ml, SCF 50ng/ml, Epo 2U/ $\mathrm{ml}$, G-CSF $6.36 \mathrm{ng} / \mathrm{ml}$, GM-CSF 50ng/ml). After 14 days, colonies were counted under an inverted microscopy.

\section{NOD/SCID repopulation assay}

A total of $8 \times 10^{6} \mathrm{CD} 34^{+}$cells were infected with HD-Ad5/35.Tet-2 at an MOI of 8,000 vp/cell. $100 \mathrm{ug} / \mathrm{ml}$ Dox was added after infection to induce GFP expression. 48 hours after induction, GFP positive and negative cells were sorted by FACS. A total of $3 \times 10^{5}$ cells from each fraction were transplanted into sublethally irradiated NOD-SCID (NOD-scid IL2Rgamma<null $>$ ) mice (The Jackson Laboratory, Bar Harbor, Maine). Bone marrow aspirates from femurs were obtained 4 weeks after transplantation and analyzed for engrafted human cells using antibodies specific to human CD34, CD45, CD15, CD33 and CD19 (BD Biosciences, San Jose, CA). For each staining, isotype-matched control $\mathrm{mAbs}$ were used to establish background staining levels.

For assessing the human primitive progenitor cells with colony-forming capacity in the NOD/ SCID mouse bone marrow, a total of 75,000 bone marrow cells from each mouse were plated and subjected to colony assays as described above. Bone marrow cells from nontransplanted NOD/SCID mice were included as negative controls to ensure the human origin of the colonies.

\section{Results}

We first wished to prove our hypothesis that HD-Ad5/35 vectors affect the clonogeneic capacity of transduced CD34+ ${ }^{+}$cells less than first-generation Ad5/35 vectors. We used previously constructed HD-Ad5/35 and first-generation Ad5/35 vectors that both contained a CMV-GFP expression cassette $[4,10]$ and infected umbilical cord blood (UBC)-derived CD34 ${ }^{+}$cells at increasing MOIs (Figs.1A and B). Cell viability and GFP fluorescence was measured by flow cytometry 48 hours after infection. While cell viability was not affected after infection with the HD-Ad5/35.GFP, the first-generation Ad5/35.GFP vector caused dosedependent toxicity at MOIs higher than $4000 \mathrm{vp} / \mathrm{cell}$ (Fig.1A). The percentage of GFP expressing cells reached a plateau after infection with HD-Ad5/35.GFP at MOIs 8000 vp/cell. In contrast, Ad5/35-GFP killed transduced cells resulting in a decline of the percentage of GFPpositive cells after infection with MOIs higher than $4000 \mathrm{vp} / \mathrm{cell}$ (Fig.1B). We then tested the effect of HD-Ad5/35.GFP and Ad5/35.GFP infection on colony formation. Forty-eight hours after infection at an MOI of $8000 \mathrm{vp} / \mathrm{cell}$, cells were sorted for GFP expression and GFP positive cells and mock-infected cells were plated in clonogenic progenitor assays (Fig.1C). The number of colonies from HD-Ad5/35 transduced cells was comparable to mock-infected $\mathrm{CD} 34^{+}$cells, indicating the absence of cytotoxicity. The number of colonies formed from cells 
transduced with first-generation Ad5/35 vector was about 3-fold lower and there was pronounced toxicity in erythroid progenitors (burst-forming unit-erythroid, BFU-E).

We constructed two types of HD-Ad5/35 vector for doxycyclin (Dox)-inducible GFP expression (Fig.2A). The first vector (HD-Ad5/35.Tet-1) contained a fusion between the Krüppel-associated box (KRAB) domain and the tetracycline repressor (TetR). In this system, tTR-KRAB-mediated repression of a PGK promoter, juxtaposed to Tet operator sequences can be reversibly controlled by Dox [22]. The second vector (HD-Ad5/35.Tet-2) contained the standard Tet-on expression system, using an Tet transactivator (tTA) for regulated transgene expression [23]. In both vectors, the GFP expression cassette is flanked by polyadenylation signals and a chromatin insulator derived from the chicken globin locus (cHS-4) to avoid unspecific interaction with enhancers and promoters present in the Ad ITR/packaging signal and stuffer DNA. HD-Ad5/35 vector titers were determined by quantitative Southern blot analysis (Fig.2B). Titers for HD-Ad5/35.Tet- 1 were $3.8 \times 10^{11} \mathrm{vp} / \mathrm{ml}$, titers for HD-

Ad5/35.Tet-2 were $5.8 \times 10^{11} \mathrm{vp} / \mathrm{ml}$. Helper virus contamination was assessed by qPCR using primers specific to the HD and helper virus genomes. In final vector preparations helper virus contamination was less than $0.5 \%$ (based on RT- PCR) or less than $0.02 \%$ (based on titering for pfu). Because of the presence of two copies of the cHS4 insulator in the vector genome, we were concerned about potential genomic rearrangements within the vector (through recombination between the homologous $1.2 \mathrm{~kb}$ cHS-4 regions) during virus replication/ amplification. We therefore analyzed vector DNA isolated from purified Ad particles (Fig.2C). Restriction enzyme analysis did not show detectable undesired genomic recombination and helper virus contamination in our final vector preparations.

We studied the properties of our HD-Ad5/35 vectors in Mo7e cells, a growth-factor dependent CD $34^{+}$erythroleukemia cell line that is often used as a model for erythroid progenitor cells [27]. Cells were infected with HD-Ad5/35.Tet- 1 and HD-Ad5/35.Tet-2 at increasing MOIs, and the percentage of GFP expressing cells as well as mean GFP fluorescence levels were measured 24 hours later in the presence and absence of $100 \mathrm{ng} / \mathrm{ml}$ Dox (Fig.3). Preliminary studies showed that no significant increase in induction of GFP expression levels was achieved with Dox concentrations higher than 100ng/ml for both HD-Ad5/35 vectors (data not shown). In Mo7e cells infected with HD-Ad5/35.Tet-1, non-induced GFP expression remained at background levels up to an MOI of $400 \mathrm{vp} / \mathrm{cell}$ (Fig.3A). At this MOI, addition of Dox induced GFP expression in nearly $100 \%$ of cells, however, the levels of GFP expression were only 8 fold higher than background (Fig.3B). For HD-Ad5/35.Tet-2 infected cells, GFP expression without induction could be detected at all MOI tested (Fig.3C). The problem of leaky expression from HD-Ad5/35.Tet-2 was particularly pronounced after infection at MOIs above $400 \mathrm{vp} / \mathrm{cell}$ (Fig.3D). At an MOI of $400 \mathrm{vp} / \mathrm{cell}$, the induction factor of GFP expression was about 200. In summary, HD-Ad5/35.Tet- 1 allowed for more tightly controlled, but less inducible GFP expression than HD-Ad5/35.Tet-2.

We next tested induction of gene expression in primary UCB-derived CD34 ${ }^{+}$cells (Fig.4). Infection with HD-Ad5/35.Tet-1, in the presence of $100 \mathrm{ng} / \mathrm{ml}$ Dox, resulted in a MOIdependent increase in GFP expressing cells (Figs.4A,B). At an MOI of $8000 \mathrm{vp} / \mathrm{cell}$, in the presence of Dox, more then 50\% of cells expressed GFP, although at a low level. In the absence of Dox, GFP expression was at background levels (Fig. 4C). Although the induction factor was low, the GFP expressing CD $34^{+}$cell fraction can clearly be distinguished by flow cytometry (Fig.4C). GFP expression levels increased with increasing Dox concentrations from 25 to $100 \mathrm{ng} / \mathrm{ml}$ (Fig.4D). Infection of CD34+ cells with HD-Ad5/35.Tet-2 at $8000 \mathrm{vp} / \mathrm{cell}$, in the presence of Dox, resulted in induced GFP expression in less than $3 \%$ of $\mathrm{CD} 34^{+}$cells (Figs. 4E,G), whereby GFP mean fluorescence levels from HD-Ad5/35.Tet-2 were about 100-fold higher than from HD-Ad5/35.Tet-1 (Figs.4F,G). Furthermore, there was detectable GFP expression without induction. Similar to HD-Ad5/35.Tet-1, we observed a dose-dependent 
increase of GFP expression levels in the range of 5 to $75 \mathrm{ng} / \mathrm{ml}$ Dox (Fig.4H). No reduction of viability was observed after infection with HD-Ad5/35.Tet- 1 and HD-Ad5/35.Tet-2 at an MOI of $8000 \mathrm{vp} / \mathrm{cell} 48$ hours p.i., compared to mock-infected cells.

The dose-response curve after infection with HD-Ad5/35.Tet-2 indicates that this expression system is active in only a specific subset (less than $3 \%$ ) of $\mathrm{CD}^{+} 4^{+}$cells. To test whether HDAd5/35 vectors mediated inducible GFP expression in subsets that contained potential HSC, we performed colony assays and NOD/SCID repopulation assays with transduced $\mathrm{CD} 34^{+}$cells. CD34 ${ }^{+}$cells were infected at an MOI of $8000 \mathrm{vp} /$ cell with HD-Ad5/35.Tet- 1 and HDAd5/35.Tet-2 in the presence of 100ng/ml Dox, and 48 hours later, GFP-positive and GFPnegative cells were sorted and plated for colony assays or transplanted into sublethally irradiated NOD-SCID mice. Because our main research focus is globin gene therapy, we focused in colony assays on the analysis of erythroid progenitor colonies (BFU-E and mixed BFU-E/CFU-GM) (Fig.5A). (There was no significant difference in the total number of colonies between the groups.) For both vectors, more mixed colonies were found in GFPpositive than GFP-negative fractions, underscoring that HD-Ad5/35 vectors target potential HSC. Interestingly, the percentage of BFU-E and mixed colonies cells with induced GFP expression was significantly higher for HD-Ad5/35.Tet-2 than for HD-Ad5/35.Tet-1 ( $\mathrm{p}<0.01)$. Overall, both expression systems appear to allow for induced GFP expression in clonogenic progenitors, whereby HD-Ad5/35-Tet-2 has higher background expression without induction, similar to what we observed for Mo7e cells. To further confirm that the small fraction of GFP positive cells resulting from HD-Ad5/35.Tet-2 transduction contains HSC, we performed NOD/SCID repopulation assays with $\mathrm{GFP}^{+}$and $\mathrm{GFP}^{-} \mathrm{CD} 34^{+}$cells (Fig.5B). Analysis of bone marrow 4 weeks after transplantation showed that both $\mathrm{GFP}^{+}$and $\mathrm{GFP}^{-}$fractions reconstituted sublethally irradiated animals with both lymphoid and myeloid human cell contributions. This underscores the absence of cytotoxicity associated with HD-Ad5/35 transduction. To assess whether $\mathrm{GFP}^{+} \mathrm{NOD} / \mathrm{SCID}$ repopulating cells were capable of generation of primitive hematopoietic progenitor cells in NOD/SCID mouse bone marrow, we plated mouse bone marrow cells in methylcellulose medium with cytokines specifically supporting the growth of human progenitor cells (Fig.5C). This study showed that bone marrow cells from recipients transplanted with $\mathrm{CD} 34^{+} \mathrm{GFP}^{+}$cells did contain CFU-GM and BFU-E. We observed no reduction in the numbers of colony-forming cells between the recipients transplanted with the $\mathrm{CD} 4^{+} \mathrm{GFP}^{+}$or $\mathrm{GFP}^{-}$fractions, however there were more BFU-E in mice transplanted with $\mathrm{GFP}^{+}$fractions. Similar NOD/SCID repopulation rates were achieved with $\mathrm{GFP}^{+}$cells after HD-Ad5/35.Tet-1 transduction (data not shown).

In summary, HD-Ad5/35-Tet-2 confers inducible GFP expression in a restricted subset of $\mathrm{CD} 34^{+}$cells capable of repopulating NOD/SCID mice.

\section{Discussion}

Inducible gene expression from a non-integrating, non-toxic HD-Ad5/35 vector in HSC has a series of potential applications in stem cell biology and gene therapy. We focused our attempts on incorporating the Tet-inducible expression systems into HD-Ad5/35 vectors. Two variants of the Tet-systems have been widely used [23]. In the original version, Tet-off, the Tet transactivator in the absence of Dox binds to Tet responsive elements (TetO) and activates transcription. The Tet-on system employs a mutated reverse transactivator rtTA, which binds to TetO and activates transcription in the presence of Dox.

Previous studies indicated leakiness of both these systems in the context of Ad vectors, due to unspecific activation of the TetO/minimal CMV promoter by viral promoters and enhancers [16] [17]. In our HD-Ad5/35 vectors the Tet-inducible expression cassettes are flanked by Ad ITRs, the Ad packaging signal, and stuffer DNA derived from human X chromosome [28]. 
The stuffer DNA was fragmented and scrambled to avoid large stretches of homology with human sequences, and potential integration through homologous recombination. Although no obvious canonical promoter or enhancer sequences could be identified in the stuffer DNA, interference with the Tet-inducible transcription system cannot be excluded. To address this problem, we flanked our expression cassette with i) polyadenylation signals that are able to block read-through transcription from promoters contained in the Ad ITRs [12] and, potentially, in the stuffer DNA sequence, and ii) cHS4-derived insulators that are able to block unspecific trans-activation of the TetO/minCMV promoter by Ad and stuffer enhancers (including the E1A enhancer that partially overlaps with the Ad packaging signal). We have previously shown that the incorporation of cHS4 insulators greatly improved inducible [18] and tissue specific gene expression [19] from Ad vectors.

A second new feature described in this study is the use of a tTR-KRAB repressor in the context of HD-Ad vectors. Binding of DNA binding proteins fused to KRAB results in histone deacetylation and methylation, thus creating a local heterochromatin state and inactivation of promoters that are 2 to $3 \mathrm{~kb}$ up- or downstream of the binding site [29]. It has been shown that tTR-KRAB mediated repression of cellular Pol-II and Pol-III promoters juxtaposed to the TetO can be reversibly controlled by Dox [22]. These studies were done with transgenes cassettes integrated into the cell genome. After infection, Ad genomes remain episomal, but within hours they lose their packaging by Ad DNA binding protein, and become associated with cellular histones and non-histone proteins, converting it into a nucleosome-like structure [30,31]. We therefore hypothesized that the tTR-KRAB system would also work in the context of episomal Ad genomes. We were also interested in this system because it might allow for combining tight, inducible and tissue-specific expression by, for example, replacing the PGK promoter with a tissue-specific promoter. Overall, we found that the TTR-KRAB system when incorporated into HD-Ad5/35 (HD-Ad5/35.Tet-1) represses gene expression better when not induced than the standard rtTA system (HD-Ad5/35.Tet-2). This feature might be critical for expression of proteins with endonuclease activity such as AAV Rep78 or zinc-finger nucleases, because trace amounts of these proteins appear to be toxic to producer cells or interfere with Ad replication ([32], unpublished observations). When compared with HD-Ad5/35.Tet-2, the tTR-KRAB based HD-Ad5/35.Tet-1 vector system conferred lower expression levels when induced in Mo7e and $\mathrm{CD} 34^{+}$cells. This might not necessarily be a disadvantage because biologically active proteins in HSC are often needed only at low concentrations. For both HDAd5/35.Tet- 1 and Tet-2, transgene expression can be titrated by varying Dox concentrations.

Interestingly, in CD34+ cells, while HD-Ad5/35.Tet-1 allowed for activated gene expression in all transduced cells ( $\sim 50 \%$ at an MOI $8000 \mathrm{vp} / \mathrm{cell})$, induced gene expression from HD-

Ad5/35.Tet- 2 was restricted to a relatively small subset of CD34\% cells ( $<3 \%$ at an MOI of $8,000 \mathrm{vp} / \mathrm{cell}$ ), and the percentage of transduced cells did not increase further at higher MOIs. Importantly, clonogenic assays and repopulation assays in NOD/SCID mice showed that both HD-Ad5/35.Tet- 1 and -2 vectors allow for induced gene expression in hematopoietic progenitor/stem cells. These assays also confirm that transduction of $\mathrm{CD} 34^{+}$cells with HDAd5/35 is not associated with cytotoxity, a problem encountered with first-generation Ad5/35 vectors.

In summary, we present two versatile HD-Ad5/35 vector expression systems that allow for tightly regulated transgene expression in human HSC. Our current efforts are focused on the construction of HD-Ad5/35 vectors for inducible expression of Rep78, Zinc-finger nucleases, and meganucleases.

\section{Acknowledgements}

We thank Daniel Stone for editing the manuscript. This study was supported by NIH grant P01 HL53750. 


\section{References}

1. Wang H, Lieber A. A helper-dependent capsid-modified adenovirus vector expressing adenoassociated virus rep78 mediates site-specific integration of a 27-kilobase transgene cassette. J Virol 2006;80:11699-11709. [PubMed: 16987973]

2. Miller JC, Holmes MC, Wang J, et al. An improved zinc-finger nuclease architecture for highly specific genome editing. Nat Biotechnol 2007;25:778-785. [PubMed: 17603475]

3. Yanez-Munoz RJ, Balaggan KS, MacNeil A, et al. Effective gene therapy with nonintegrating lentiviral vectors. Nat Med 2006;12:348-353. [PubMed: 16491086]

4. Shayakhmetov DM, Papayannopoulou T, Stamatoyannopoulos G, Lieber A. Efficient gene transfer into human CD34(+) cells by a retargeted adenovirus vector. J Virol 2000;74:2567-2583. [PubMed: 10684271]

5. Gaggar A, Shayakhmetov D, Lieber A. CD46 is a cellular receptor for group B adenoviruses. Nature Medicine 2003;9:1408-1412.

6. Yotnda P, Onishi H, Heslop HE, et al. Efficient infection of primitive hematopoietic stem cells by modified adenovirus. Gene Ther 2001;8:930-937. [PubMed: 11426333]

7. Nilsson M, Karlsson S, Fan X. Functionally distinct subpopulations of cord blood CD34+ cells are transduced by adenoviral vectors with serotype 5 or 35 tropism. Mol Ther 2004;9:377-388. [PubMed: 15006604]

8. Lieber A, He CY, Kirillova I, Kay MA. Recombinant adenoviruses with large deletions generated by Cre-mediated excision exhibit different biological properties compared with first-generation vectors in vitro and in vivo. J Virol 1996;70:8944-8960. [PubMed: 8971024]

9. Kochanek S, Schiedner G, Volpers C. High-capacity 'gutless' adenoviral vectors. Curr Opin Mol Ther 2001;3:454-463. [PubMed: 11699889]

10. Wang H, Shayakhmetov DM, Leege T, et al. A capsid-modified helper-dependent adenovirus vector containing the beta-globin locus control region displays a nonrandom integration pattern and allows stable, erythroid-specific gene expression. J Virol 2005;79:10999-11013. [PubMed: 16103151]

11. Balamotis MA, Huang K, Mitani K. Efficient delivery and stable gene expression in a hematopoietic cell line using a chimeric serotype 35 fiber pseudotyped helper-dependent adenoviral vector. Virology 2004;324:229-237. [PubMed: 15183069]

12. Hearing P, Shenk T. The adenovirus type 5 E1A transcriptional control region contains a duplicated enhancer element. Cell 33:695-703. [PubMed: 6871991]

13. Grable M, Hearing P. cis and trans requirements for the selective packaging of adenovirus type 5 DNA. J Virol 1992;66:723-731. [PubMed: 1731109]

14. Shi Q, Wang Y, Worton R. Modulation of the specificity and activity of a cellular promoter in an adenoviral vector. Hum Gene Ther 1997;8:403-410. [PubMed: 9054515]

15. Friedman JM, Babiss LE, Clayton DF, Darnell JE Jr. Cellular promoters incorporated into the adenovirus genome: cell specificity of albumin and immunoglobulin expression. Mol Cell Biol 1986;6:3791-3797. [PubMed: 2948108]

16. Xu ZL, Mizuguchi H, Mayumi T, Hayakawa T. Regulated gene expression from adenovirus vectors: a systematic comparison of various inducible systems. Gene 2003;309:145-151. [PubMed: 12758130]

17. Salucci V, Scarito A, Aurisicchio L, et al. Tight control of gene expression by a helper-dependent adenovirus vector carrying the rtTA2(s)-M2 tetracycline transactivator and repressor system. Gene Ther 2002;9:1415-1421. [PubMed: 12378403]

18. Steinwaerder DS, Lieber A. Insulation from viral transcriptional regulatory elements improves inducible transgene expression from adenovirus vectors in vitro and in vivo. Gene Ther 2000;7:556567. [PubMed: 10819570]

19. Ye X, Liang M, Meng X, et al. Insulation from viral transcriptional regulatory elements enables improvement to hepatoma-specific gene expression from adenovirus vectors. Biochem Biophys Res Commun 2003;307:759-764. [PubMed: 12878174]

20. Majem M, Cascallo M, Bayo-Puxan N, Mesia R, Germa JR, Alemany R. Control of E1A under an E2F-1 promoter insulated with the myotonic dystrophy locus insulator reduces the toxicity of oncolytic adenovirus Ad-Delta24RGD. Cancer Gene Ther 2006;13:696-705. [PubMed: 16498429] 
21. Hallenbeck PL, Chang YN, Hay C, et al. A novel tumor-specific replication-restricted adenoviral vector for gene therapy of hepatocellular carcinoma. Hum Gene Ther 1999;10:1721-1733. [PubMed: 10428217]

22. Szulc J, Wiznerowicz M, Sauvain MO, Trono D, Aebischer P. A versatile tool for conditional gene expression and knockdown. Nat Methods 2006;3:109-116. [PubMed: 16432520]

23. Gossen M, Freundlieb S, Bender G, Muller G, Hillen W, Bujard H. Transcriptional activation by tetracyclines in mammalian cells. Science 1995;268:1766-1769. [PubMed: 7792603]

24. Wang H, Shayakhmetov DM, Leege T, et al. A Capsid-Modified Helper-Dependent Adenovirus Vector Containing the $\{$ beta $\}$-Globin Locus Control Region Displays a Nonrandom Integration Pattern and Allows Stable, Erythroid-Specific Gene Expression. J Virol 2005;79:10999-11013. [PubMed: 16103151]

25. Hartigan-O'Connor D, Barjot C, Salvatori G, Chamberlain JS. Generation and growth of gutted adenoviral vectors. Methods Enzymol 2002;346:224-246. [PubMed: 11883070]

26. Barjot C, Hartigan-O'Connor D, Salvatori G, Scott JM, Chamberlain JS. Gutted adenoviral vector growth using E1/E2b/E3-deleted helper viruses. J Gene Med 2002;4:480-489. [PubMed: 12221641]

27. Avanzi GC, Lista P, Giovinazzo B, et al. Selective growth response to IL-3 of a human leukaemic cell line with megakaryoblastic features. Br J Haematol 1988;69:359-366. [PubMed: 3261598]

28. Sandig V, Youil R, Bett AJ, et al. Optimization of the helper-dependent adenovirus system for production and potency in vivo. Proc Natl Acad Sci U S A 2000;97:1002-1007. [PubMed: 10655474]

29. Moosmann P, Georgiev O, Thiesen HJ, Hagmann M, Schaffner W. Silencing of RNA polymerases II and III-dependent transcription by the KRAB protein domain of KOX1, a Kruppel-type zinc finger factor. Biol Chem 1997;378:669-677. [PubMed: 9278146]

30. Tate VE, Philipson L. Parental adenovirus DNA accumulates in nucleosome-like structures in infected cells. Nucleic Acids Res 1979;6:2769-2785. [PubMed: 461205]

31. Mirza MA, Weber J. Structure of adenovirus chromatin. Biochim Biophys Acta 1982;696:76-86. [PubMed: 7082670]

32. Carlson CA, Shayakhmetov DM, Lieber A. An Adenoviral Expression System for AAV Rep78 Using Homologous Recombination. Mol Ther 2002;6:91-98. [PubMed: 12095308] 
A

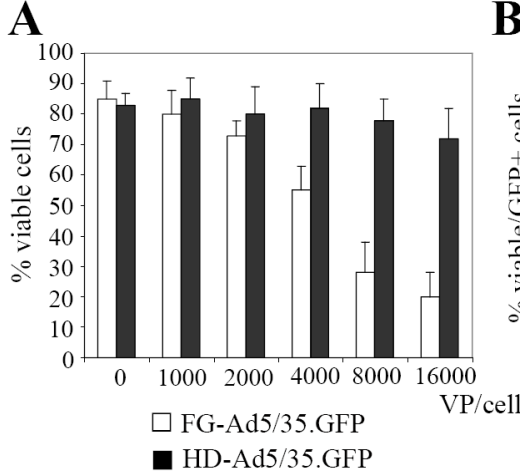

B

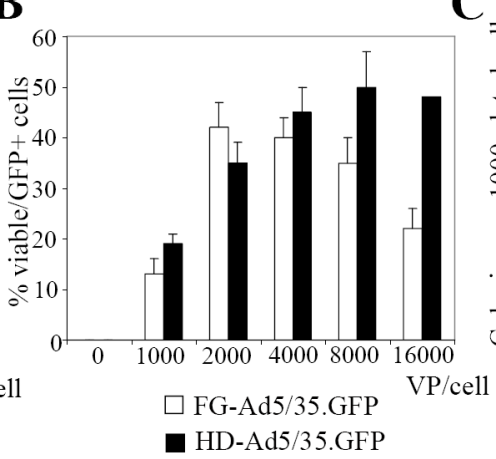

$\mathrm{C}$

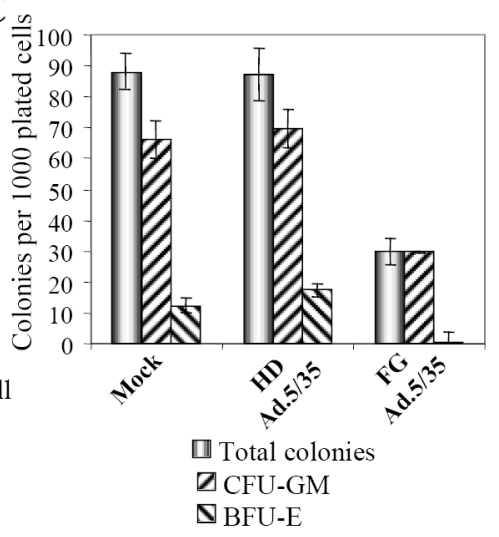

Fig. 1. Effect of Ad vector infection on cell viability and clonogenic capacity of $\mathrm{CD}^{+}{ }^{+}$cells $A$ and B) Human UBC-derived CD34+ cells were mock-infected or infected with an HDAd5/35 vector or first-generation (FG)-Ad5/35 vector at increasing MOIs with helperdependent HD-Ad5/35.GFP and first-generation FG-Ad5/35.GFP vectors. Cell viability and transduction efficiency was measured 48 hours later by flow cytometry for PI and GFP fluorescence respectively.

C) CD34+ cells were infected with HD-Ad5/35.GFP or FG-Ad5/35.GFP at an MOI of 8000 $\mathrm{vp} / \mathrm{cell}$. Forty-eight hours after infection, GFP positive cells from Ad infected samples were sorted by flow cytometry and subjected to clonogenic assays in comparison to mock-infected cells. The numbers of total colonies, BFU-E and CFU-GM per 1000 plated cells were counted 14 days after seeding the cells. $\mathrm{N}=3$

CFU-GM: Cells with colony-forming unit-granulocyte-macrophage capacity

BFU-E: Cells with burst-forming unit-erythroid capacity

Mixed: Primitive cells with colony-forming unit-granulocyte, erythrocyte, macrophage, and megakaryocyte capacity

Shown are Mean values +/- SEM. 


\section{A}

HD-Ad5/35.Tet-1(28.2kb)

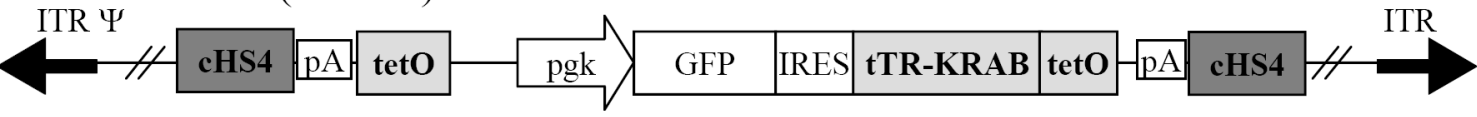

HD-Ad5/35.Tet-2(28.5kb)

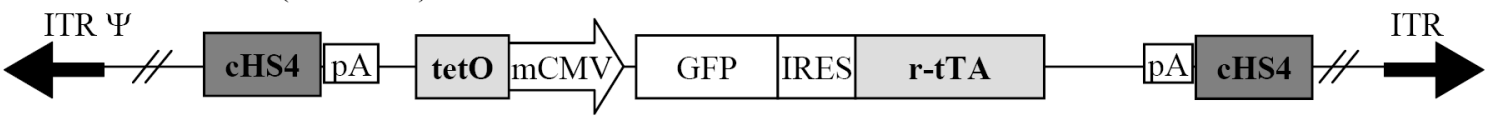

B

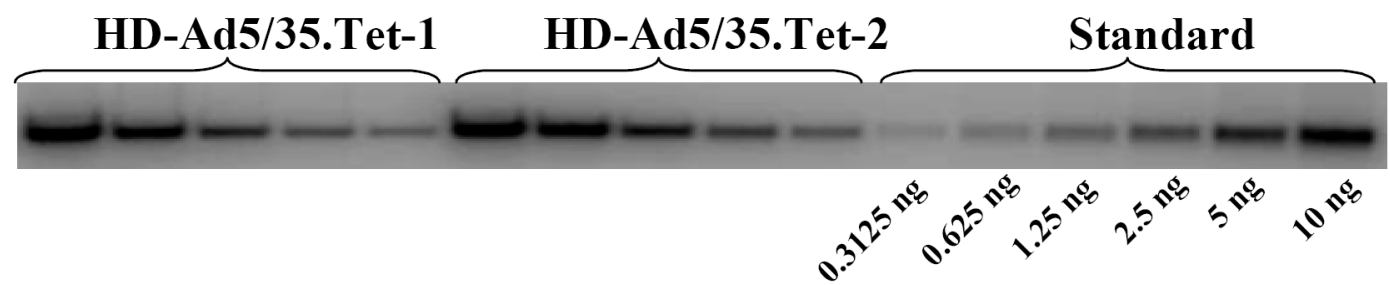

C
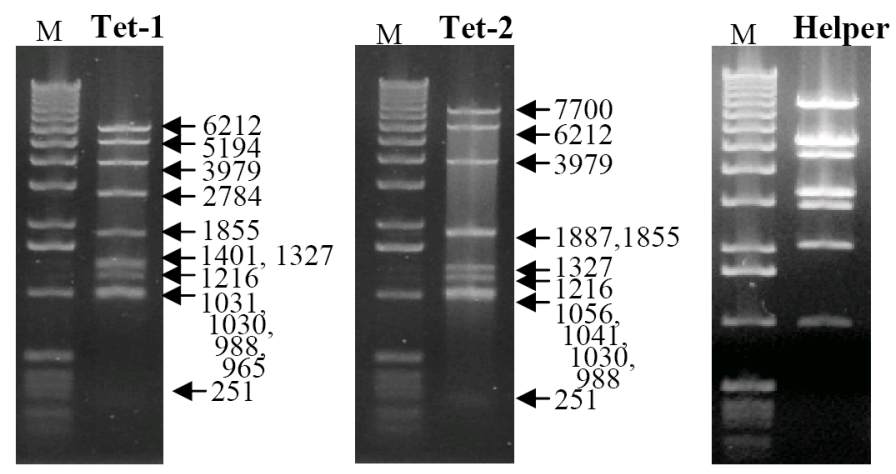

Fig. 2. HD-Ad5/35 vectors containing Tet inducible expression systems

A) Vector schemes: Ad5/35.Tet-1 contained a fusion between the Krüppel-associated box (KRAB) domain and the tetracycline repressor (TetR). A bicitronic GFP-tTR-KRAB cassette is driven be a PGK promoter. Two Tet operator (TetO) sequences flank this expression cassette. In non-induced stage tTR-KRAB binds to the TetO and blocks expression. Addition of Dox removes the repressor. HD-Ad5/35. Tet- 2 contains the standard Tet-on expression system. In both vectors the GFP expression cassette is flanked by polyadenylation signals (pA) and chicken globin locus (cHS-4) chromatin insulators.

B). Quantitative Southern blot analysis of vector titers. As a standard, pHD-Ad.Tet-2 was linearized with PmeI and diluted serially corresponding to $0.3125 \mathrm{ng}, 0.625 \mathrm{ng}, 1.25 \mathrm{ng}, 2.5$ $\mathrm{ng}, 5 \mathrm{ng}, 10 \mathrm{ng}$. A $20 \mu \mathrm{l}$ aliquot of purified HD virus stock mixed with $180 \mu \mathrm{l}$ PBS plus $2 \times$ $10^{5} 293$ cells (as a source of carrier DNA) was subjected to pronase digestion, subsequent 
phenol-chloroform purification, and ethanol precipitation. Serially diluted, extracted viral DNAs were run on an agarose gel together with the standard curve and analyzed by Southern blotting and phosphor-imaging as described earlier [10].

C) HindIII restriction pattern after digestions of vector DNA from purified HD-Ad5/35.Tet-1 (left panel), HD-Ad5/35.Tet-2 (middle panel) and Ad5/35 helper virus (right panel). 
HD-Ad5/35.Tet-1

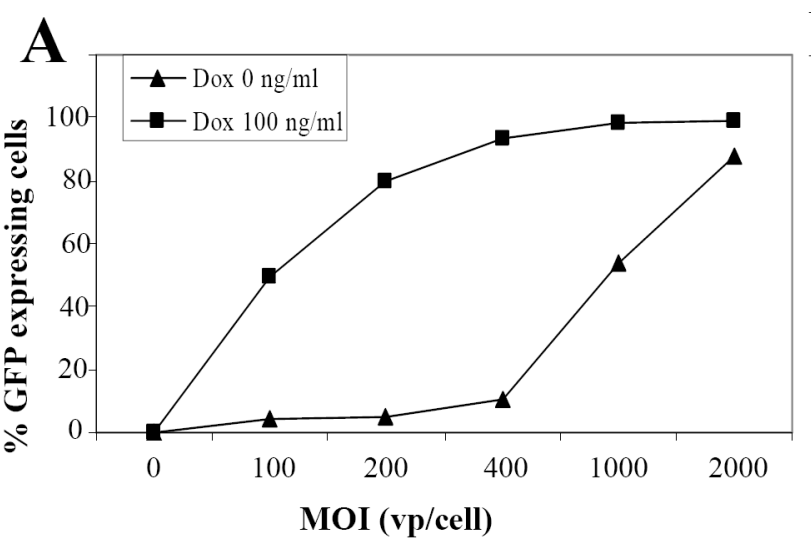

B

HD-Ad5/35.Tet-2
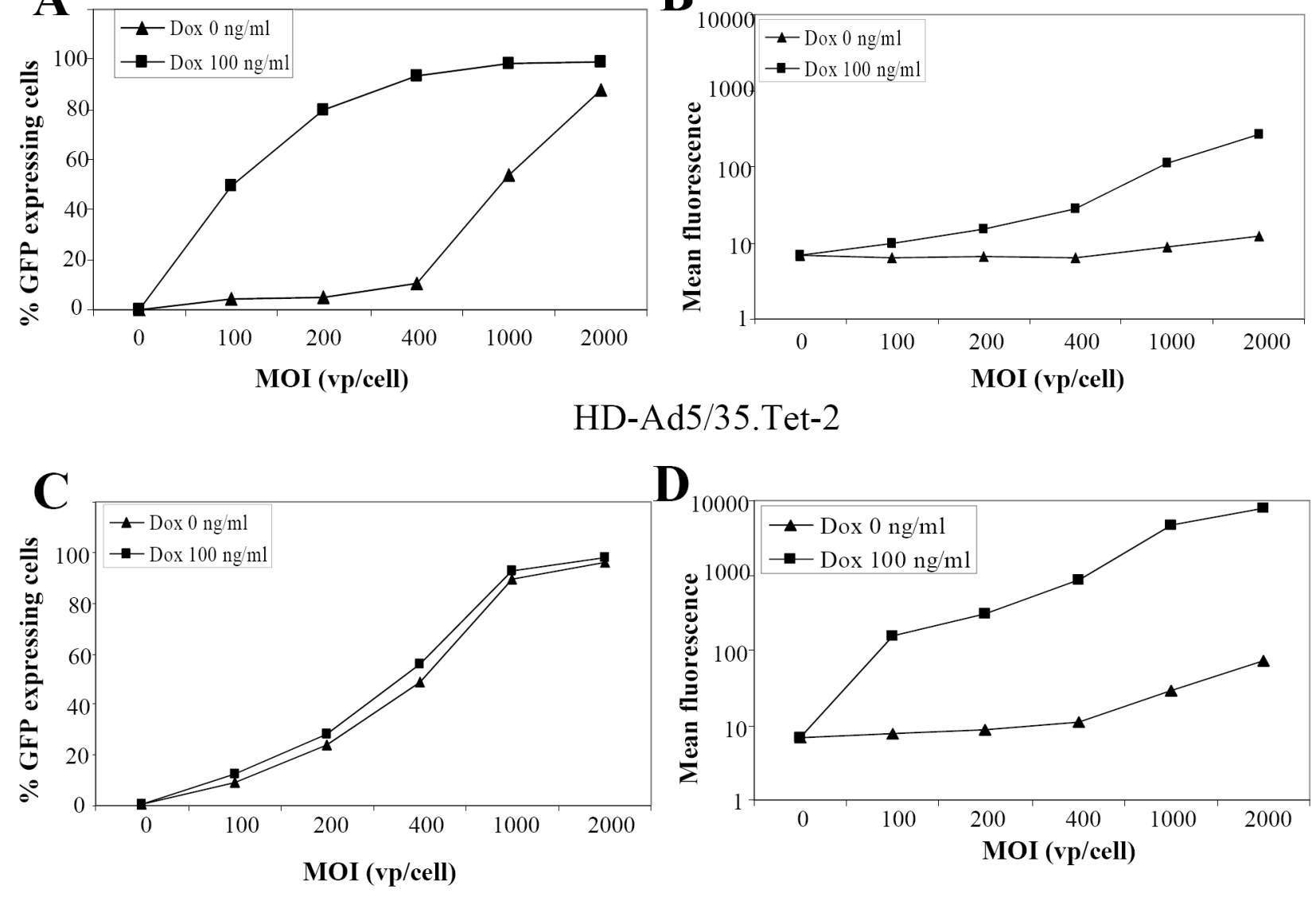

D

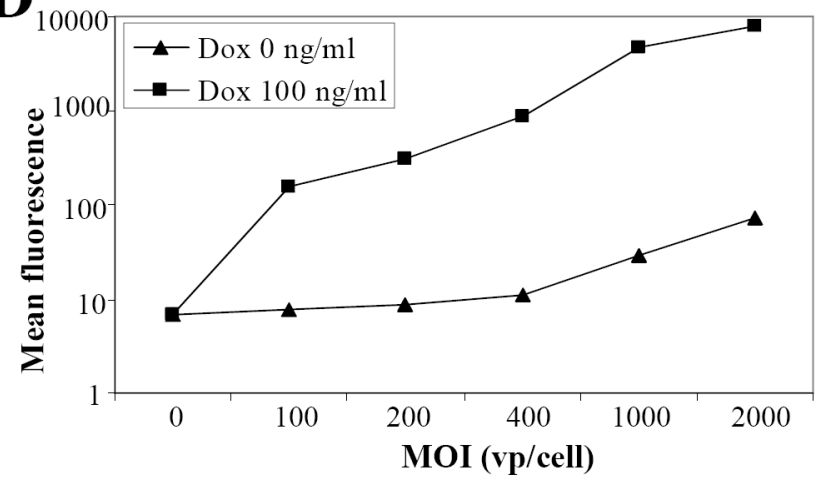

Fig. 3. Transduction studies in Mo7e cells

MO7e cells were infected with HD-Ad5/35.Tet-1 (A,B) or HD-Ad5/35.Tet-2 (C,D) at increasing MOIs of 100, 200, 400, 1000, $2000 \mathrm{vp} / \mathrm{cell}$. One set of infected cells was left noninduced, to the other set of cells, $100 \mathrm{ng} / \mathrm{ml}$ Doxycyclin were added for induction. Twenty-four hours after infection/induction, the percentage of GFP expressing cells $(A, C)$ and mean GFP fluorescence $(B, D)$ were measured by flow cytometry.

$\mathrm{N}=3$. The SEM was consistently less than $10 \%$ of the Mean. 


\section{HD-Ad5/35.Tet-1}
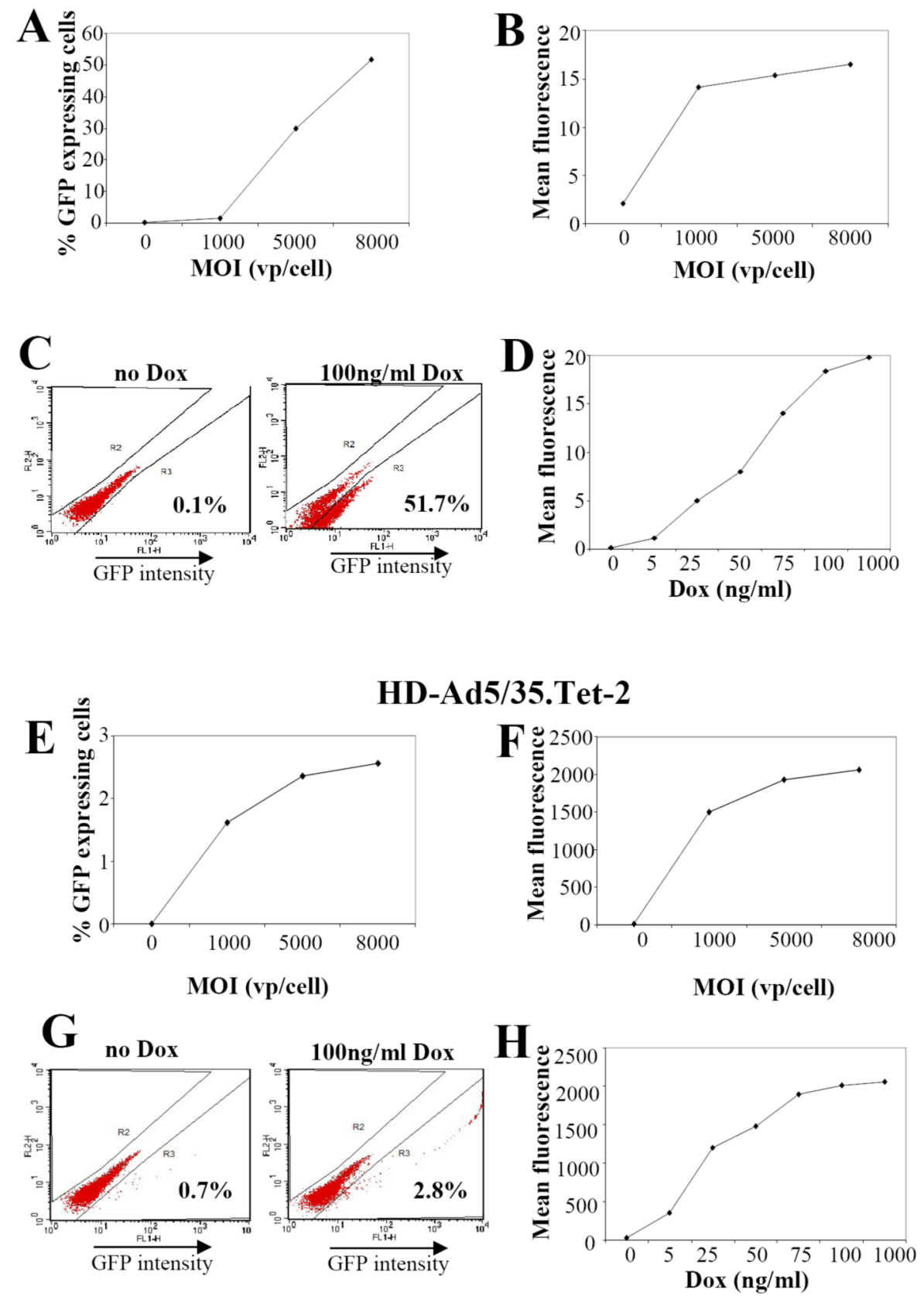

Fig. 4. Transduction studies in human $\mathrm{CD34}^{+}$cells

UBC-derived human CD34+ cells were infected with HD-Ad5/35.Tet-1 (A-D) or HDAd5/35.Tet-2 (E-H) and 100ng/ml Dox was added after infection to induce expression. Fortyeight hours post-induction, GFP expression was analyzed by flow cytometry.

$\mathrm{CD} 34^{+}$cells were infected at increasing MOI and the percentage of GFP expressing cells (A, E) and mean GFP fluorescence (B, F) were measured.

$\mathrm{CD} 4^{+}$cells were infected at an MOI of $8000 \mathrm{vp} / \mathrm{cell}$ and GFP expressing was analyzed without induction and in the presence of 100ng/ml of Dox 48 hours post-infection/induction $(\mathrm{C}, \mathrm{G})$ $\mathrm{CD} 4^{+}$cells were infected at an MOI of $8000 \mathrm{vp} / \mathrm{cell}$ and incubated with the indicated concentrations of Dox (D,H). Mean GFP fluorescence was measured 48 hours later. 
$\mathrm{N}=3$. The SEM was consistently less than $10 \%$ of the Mean. 
A

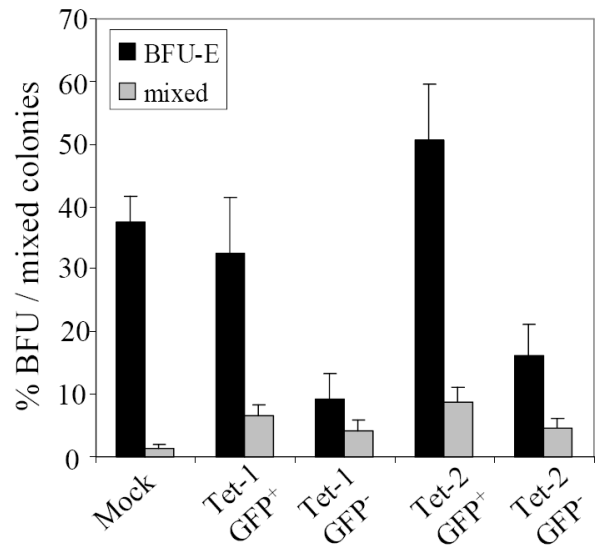

B

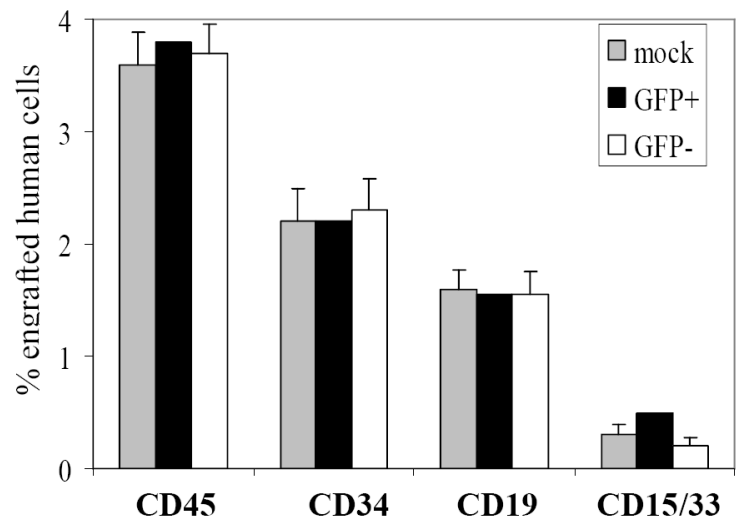

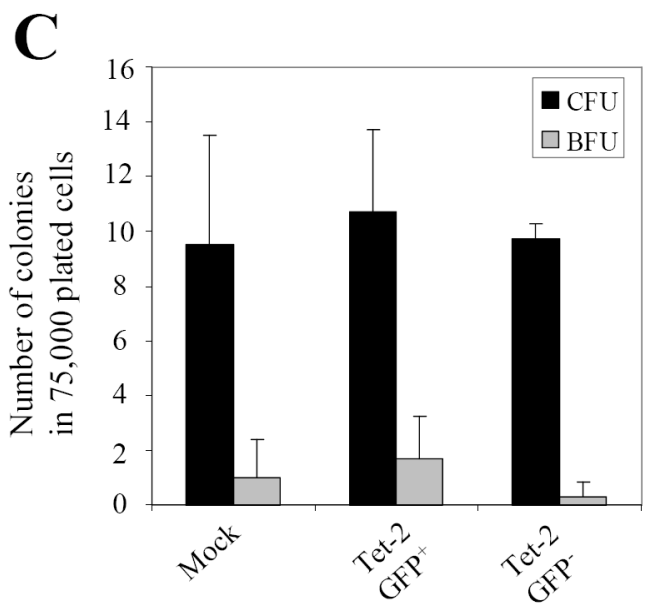

Fig. 5. Analysis of stem cells capacity of GFP expressing $\mathrm{CDB4}^{+}$cells

UBC-derived human CD34+ cells were mock-infected or infected with HD-Ad5/35.Tet-1

(Tet-1) or HD-Ad5/35.Tet-2 (Tet-2) at an MOI of $8000 \mathrm{vp} / \mathrm{cell}$ and 100ng/ml Dox was added after infection to induce expression. Forty-eight hours post-induction, GFP expressing cells $\left(\mathrm{GFP}^{+}\right)$and GFP negative cells $\left(\mathrm{GFP}^{-}\right)$were sorted and either plated for colony assays (A) or used for repopulation assays in NOD/SCID mice (B). Notably infection of CD34+ cells with HD-Ad5/35 vectors at an MOI of $8000 \mathrm{vp} /$ cells is not associated cytotoxicity.

A) For colony assays, 3000 cells/plate were seeded in methylcellulose containing human growth factors. The total number of colonies, and the numbers of BFU-E and mixed colonies were counted. Shown is the percentage of colonies derived from erythroid progenitors relative to the total number of colonies in $\mathrm{GFP}^{+}$and GFP- fractions of Ad transduced $\mathrm{CD} 34^{+}$cells in comparison to mock-infected cells. $\mathrm{N}=3$

B) A total of $3 \times 10^{5}$ cells from mock-infected cells and GFP+ and GFP-cells were transplanted into sublethally irradiated NOD/SCID mice. Bone marrow aspirates from femurs were obtained 4 weeks after transplantation and analyzed for engrafted human cells.

C) Colony assays with NOD/SCID repopulating cells. A total of 75,000 cells obtained from bone marrow of NOD/SCID mice 4 weeks after transplantation of CD34 ${ }^{+}$cells transduced with HD-Ad5/35.Tet-2 were subjected to colony assays. Colonies were counted 14 days after plating. "Mock" means cells from mice that were transplanted with mock-infected human CD34+ cells. 